\title{
Food Alone May Not Provide Sufficient Micronutrients for Preventing Deficiency ${ }^{1}$
}

\author{
Bill Misner
}

Research and Product development, E-Caps \& Hammer Nutrition, Whitefish, MT. Address correspondence to drbill@e-caps.com. ${ }^{1}$ This paper was originally published in the Townsend Letter for Doctors and Patients (C) April 2005 \#261, pages 49-52. It is reproduced for JISSN, by permission, courtesy of Bill Misner, Ph.D. and the Townsend Letter for Doctors and Patients.

Received January 25, 2006/Accepted June 5, 2006

\section{ABSTRACT}

The American Dietetic Association (ADA) has stated that the best nutritional strategy for promoting optimal health and reducing the risk of chronic disease is to wisely choose a wide variety of foods. Seventy diets were computer analyzed from the menu of athletes or sedentary subjects seeking to improve the quality of micronutrient intake from food choices. All of these dietary analyses fell short of the recommended 100\% RDA micronutrient level from food alone. Therefore, based on diets analyzed for adequacy or inadequacy of macronutrients and micronutrients, a challenging question is proposed: "Does food selection alone provide $100 \%$ of the former RDA or newer RDI micronutrient recommended daily requirement?" Journal of the International Society of Sports Nutrition. 3(1):51-55, 2006

Key Words: nutrition, RDA, RDI, micronutrients

\section{INTRODUCTION}

Reference Daily Intakes (RDI) is a new term that replaces the familiar U.S. Recommended Daily Allowances (U.S. RDA). RDIs are based on a population-weighted average of the latest RDAs for vitamins and minerals for healthy Americans over 4 years old. RDIs are not recommended optimal daily intake figures for any particular age group or sex. Government-established Reference Daily Intake guidelines (RDI) are designed to prevent nutrientdeficiency diseases. Most nutritionally oriented professionals imply that a balanced variety of foods selected from the Food Guide Pyramid (FGP) will supply all micronutrients at the RDA or new RDI levels necessary to maintain optimal health and prevent nutrient-deficiency diseases. The American Dietetic Association (ADA) has proposed a conservative strategy for managing dietary micronutrient deficiency and sufficiency:

"It is the position of the American Dietetic Association (ADA) that the best nutritional strategy for promoting optimal health and reducing the risk of chronic disease is to wisely choose a wide variety of foods. Additional nutrients from fortified foods and/or supplements can help some people meet their nutritional needs as specified by science-based nutrition standards such as the Dietary Reference Intakes. This position paper addresses increasing the nutrient density of foods or diets through fortification or supplementation when diets fail to deliver consistently adequate amounts of vitamins and minerals."

Between 1996 and 2005, 70 diets were computer analyzed from the menu of athletes or sedentary subjects seeking to improve the quality of micronutrient intake from food choices. Surprisingly, all of these dietary analyses fell short of the recommended $100 \%$ RDA micronutrient level from food alone. Therefore, based on diets analyzed for adequacy or inadequacy of macronutrients and micronutrients, a challenging question is proposed:

"Does food selection alone provide $100 \%$ of the former RDA or newer RDI micronutrient recommended daily requirement?"

\section{METHODS}

From 70 computer-generated dietary analyses, 20 subjects' diets were selected based on the highest number of foods analyzed from 10 men (ages 25$50 \mathrm{y}$ ) and 10 women (ages 24-50y). A First Data Bank 
Nutritionist IV computer-program default was utilized, defaulted to apply the Harris-Benedict equation, a formula that determines energy expense against RDA micronutrient requirement, by age, gender, and body mass index (BMI). The purpose of this study was to determine if food intake alone provided the Recommended Daily Allowances (RDA) requirements for 10 vitamins and 7 minerals. The ten vitamins analyzed were Vitamin A, Vitamin D, Vitamin E, Vitamin K, Vitamin B-1, Vitamin B-2, Vitamin B-3, Vitamin B-6, Vitamin B-12, and Folate. The seven minerals analyzed were Iodine, Potassium, Calcium, Magnesium, Phosphorus, Zinc, and Selenium.

The 20 Individual Diets analyzed originated from the following subjects:

1. Two professional cyclists athletes (A)

2. Three amateur cyclists athletes (A)

3. Three amateur triathletes athletes (A)

4. Five eco-challenge amateur athletes (A)

5. One amateur runner athlete (A)

6. Six sedentary non-athletes $(\mathrm{S})$

Hence, fourteen (14) athletes' (A) and six (6) sedentary subjects' (S) diets were analyzed for calorie and RDA-micronutrient adequacy or inadequacy.

\section{RESULTS}

Based on each subject's activity level (caloric expense), age, gender, and body mass index (BMI), 10 of the diets were found calorie-excessive, above energy requirements ( 4 men and 6 women), but the remaining 10 diets were found calorie-deficient, not meeting $100 \%$ of their energy requirements $(6$ men and 4 women). When total calorie intake percents were averaged by gender, men consumed only $92.6 \%$ of the calories required for their total energy requirements, while women consumed only $97.3 \%$ of the calories required to meet their energy requirements. Of the 20 diets analyzed, $50 \%$ were calorie-sufficient and $50 \%$ calorie-deficient resulting in an overall $-7.4 \%$ deficiency for men and a $-2.7 \%$ deficiency in women (Table 1.).

Calorie deficient diets tended to record a greater number of micronutrient deficiencies as compared to the calorie-sufficient diets. Of the 340 micronutrient entries generated from 17 micronutrients analyzed,
TABLE 1. MICRONUTRIENT DEFICIENCY FROM DIETS 10 MEN \& 10 WOMEN \%

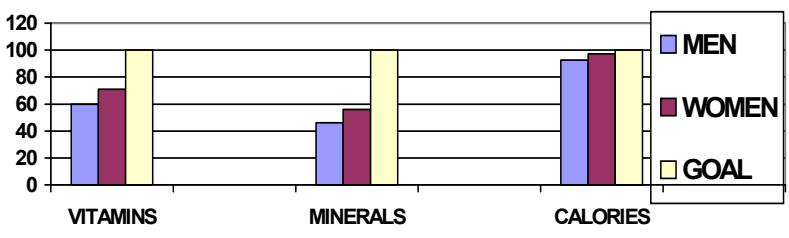

all 20 subjects presented between 3 and 15 deficiencies each based on the Recommended Daily Allowances (RDA) value from food intake alone. Males averaged deficiencies in $40 \%$ of the vitamins and $54.2 \%$ of the minerals required. Females averaged deficiencies in $29 \%$ of the vitamins and $44.2 \%$ of the minerals Recommended Daily Allowances (RDA) required. The male food intake was RDA-deficient in 78 out of 170 micronutrient entries, or $45.8 \%$ of the 10 vitamins and 7 minerals analyzed. The female dietary intake was RDAdeficient in 60 out of 170 micronutrients or $35.2 \%$ of the 10 vitamins and 7 minerals analyzed. Both male and females as a single entity recorded 138 micronutrient deficiencies out of the possible 340 micronutrients analyzed, or $40.5 \%$ micronutrient RDA-deficiency from food intake alone. (Table 2.)

\section{CONCLUSION}

Accuracy of the individual food-weighed measures, accuracy in reporting foods consumed, and the accuracy of the computer-generated software are factors that affect the accuracy of the results reported in this observational study. The effect of activity on calorie deficiency in this contingent demonstrates an increased micronutrient deficiency in athletes (A) and surprisingly, the sedentary subjects $(\mathrm{S})$ in this study also posted food-borne micronutrient deficiencies. Each chronic deficiency proportionately increases the risk of nutrient-deficiency diseases. In highly active athletes (A), micronutrient deficiencies occur at higher rates because calorie deficits are associated with exercise expense. Food alone in all 20 subjects did not meet the minimal Recommended Daily Allowances (RDA) micronutrient requirements for preventing nutrient-deficiency diseases. The more active the person, the greater the need to employ a variety of balanced micronutrient-enriched foods including micronutrient supplementation as a preventative protocol for preventing these observed deficiencies. (Tables 3, 4, 5).

Concern for micronutrient adequacy from food alone is not a new question. Excerpts 70 years ago (1936) 
from the 2nd Session of the 74th USA Congressional Record (excerpts) stated:

\begin{abstract}
"Laboratory tests prove that the fruits, the vegetables, the grains, the eggs and even the milk and the meats of today are not what they were a few generations ago (which doubtless explains how our forefathers thrived on a selection of foods that would starve us today). It is bad news to learn from our leading authorities that $99 \%$ of the American people are deficient in these minerals, and that a marked deficiency in any one of the more important minerals actually results in disease. Any upset of the balance, any considerable lack of one or another element, however microscopic the body requirement may be, and we sicken, suffer, and shorten our lives."
\end{abstract}

This twenty-subject dietary analysis study is not representative of the entire population, however the results supported by the 1936 congressional record, beg the question:

"Does food selection alone provide $100 \%$ of the former RDA or newer RDI micronutrient recommended daily requirement?"

It may be that chronic micronutrient insufficiency from food alone is more fact than fantasy. This study calls for a dietary analysis of a larger contingent of the population to determine if there is an association between chronic suboptimal RDI-micronutrient deficiency and suboptimal health disorders that may digress into disease.

Disclosure: Bill Misner Ph.D. is employed by $E$ CAPS \& HAMMER NUTRITION, a manufacturer of dietary supplements for endurance athletes.

Table 2. Total Dietary Micronutrients and Macronutrients

\begin{tabular}{|c|c|c|}
\hline TOTAL & MEN & WOMEN \\
\hline $\begin{array}{l}\text { TOTAL } \\
\text { MICRONUTRIENT } \\
\text { ENTRIES }\end{array}$ & $\begin{array}{c}170 \\
\text { (100 VITAMIN \& } 70 \text { MINERAL } \\
\text { ENTRIES) }\end{array}$ & $\begin{array}{c}170 \\
(100 \text { VITAMIN \& } 70 \\
\text { MINERAL ENTRIES) }\end{array}$ \\
\hline $\begin{array}{l}\text { TOTAL GROUP } \\
\text { INDIVIDUAL } \\
\text { MICRONUTRIENT } \\
\text { DEFICIENCIES }\end{array}$ & 78 & 60 \\
\hline $\begin{array}{c}\text { TOTAL } \\
\text { CALORIC AVERAGE } \\
\text { DEFICIENCY }\end{array}$ & $\begin{array}{c}92.6 \% \\
-7.4 \% \text { DEFICIENCY }\end{array}$ & $\begin{array}{c}97.3 \% \\
-2.7 \% \text { DEFICIENCY }\end{array}$ \\
\hline $\begin{array}{l}\text { TOTAL VITAMIN } \\
\text { DEFICIENCIES }\end{array}$ & $\begin{array}{c}40 \\
40 \% \text { DEFICIENCY }\end{array}$ & $\begin{array}{c}29 \\
29 \% \text { DEFICIENCY }\end{array}$ \\
\hline $\begin{array}{l}\text { TOTAL MINERAL } \\
\text { DEFICENCIES }\end{array}$ & $\begin{array}{c}38 \\
54.2 \% \text { DEFICIENCY }\end{array}$ & $\begin{array}{c}31 \\
44.2 \% \text { DEFICIENCY }\end{array}$ \\
\hline $\begin{array}{c}\text { TOTAL } \\
\text { MICRONUTRIENT } \\
\text { DEFICIENCY }\end{array}$ & $\begin{array}{c}78 \\
45.8 \% \text { DEFICIENCY }\end{array}$ & $\begin{array}{c}60 \\
35.2 \% \text { DEFICIENCY }\end{array}$ \\
\hline $\begin{array}{c}\text { GROUP I \& II } \\
\text { MALE \& FEMALE } \\
\text { MICRONUTRIENT } \\
\text { DEFICIENCY }\end{array}$ & \multicolumn{2}{|c|}{$40.5 \%$ DEFICIENCY } \\
\hline
\end{tabular}




\begin{tabular}{|c|c|c|c|c|c|c|c|c|c|c|}
\hline \multicolumn{11}{|c|}{$\begin{array}{c}\text { TABLE } 3 . \\
\text { Group I (5 Men, } 5 \text { Women) }\end{array}$} \\
\hline MICRONUTRIENT & \multicolumn{5}{|c|}{$\begin{array}{l}\text { MEN \% } \\
\text { Reference Daily Intake (RDI) }\end{array}$} & \multicolumn{5}{|c|}{$\begin{array}{l}\text { WOMEN \% } \\
\text { Reference Daily Intake (RDI) }\end{array}$} \\
\hline $\begin{array}{l}\text { MALE (M) } \\
\text { WOMEN (W) } \\
\text { ACTIVE (A) } \\
\text { SEDENTARY (S) }\end{array}$ & $\begin{array}{l}\text { M1 } \\
\text { (A) }\end{array}$ & $\begin{array}{l}\text { M2 } \\
\text { (A) }\end{array}$ & $\begin{array}{l}\text { M3 } \\
\text { (S) }\end{array}$ & $\begin{array}{l}\text { M4 } \\
\text { (A) }\end{array}$ & $\begin{array}{l}\text { M5 } \\
\text { (A) }\end{array}$ & $\begin{array}{l}\text { W1 } \\
\text { (A) }\end{array}$ & $\begin{array}{l}\text { W2 } \\
\text { (A) }\end{array}$ & $\begin{array}{l}\text { W3 } \\
\text { (A) }\end{array}$ & $\begin{array}{l}\text { W4 } \\
\text { (A) }\end{array}$ & $\begin{array}{l}\text { W5 } \\
\text { (A) }\end{array}$ \\
\hline $\begin{array}{l}\text { TOTAL CALORIES } \\
\text { INTAKE REPORTED }\end{array}$ & $55 \%$ & $59 \%$ & $53 \%$ & $161 \%$ & $112 \%$ & $64 \%$ & $77 \%$ & $138 \%$ & $104 \%$ & $103 \%$ \\
\hline VITAMIN A & $71 \%$ & $116 \%$ & $69 \%$ & $445 \%$ & $49 \%$ & $241 \%$ & $\begin{array}{l}1617 \\
\%\end{array}$ & $203 \%$ & $95 \%$ & $807 \%$ \\
\hline VITAMIN D & $19 \%$ & $59 \%$ & $63 \%$ & $30 \%$ & $32 \%$ & $10 \%$ & $38 \%$ & $1 \%$ & $70 \%$ & $8 \%$ \\
\hline VITAMIN E & $43 \%$ & $135 \%$ & $18 \%$ & $76 \%$ & $66 \%$ & $146 \%$ & $95 \%$ & $154 \%$ & $64 \%$ & $206 \%$ \\
\hline VITAMIN K & $19 \%$ & $311 \%$ & $11 \%$ & $122 \%$ & $61 \%$ & $18 \%$ & $511 \%$ & $44 \%$ & $78 \%$ & $155 \%$ \\
\hline VITAMIN B-1 & $71 \%$ & $103 \%$ & $140 \%$ & $290 \%$ & $183 \%$ & $64 \%$ & $168 \%$ & $334 \%$ & $389 \%$ & $264 \%$ \\
\hline VITAMIN B-2 & $69 \%$ & $95 \%$ & $67 \%$ & $209 \%$ & $342 \%$ & $77 \%$ & $146 \%$ & $183 \%$ & $382 \%$ & $183 \%$ \\
\hline VITAMIN B-3 & $155 \%$ & $87 \%$ & $64 \%$ & $294 \%$ & $123 \%$ & $117 \%$ & $267 \%$ & $256 \%$ & $270 \%$ & $214 \%$ \\
\hline VITAMIN B-6 & $90 \%$ & $144 \%$ & $55 \%$ & $137 \%$ & $77 \%$ & $81 \%$ & $231 \%$ & $143 \%$ & $180 \%$ & $213 \%$ \\
\hline VITAMIN B-12 & $99 \%$ & $156 \%$ & $123 \%$ & $193 \%$ & $357 \%$ & $119 \%$ & $140 \%$ & $78 \%$ & $99 \%$ & $328 \%$ \\
\hline FOLATE & $55 \%$ & $235 \%$ & $76 \%$ & $255 \%$ & $233 \%$ & $73 \%$ & $130 \%$ & $249 \%$ & $174 \%$ & $311 \%$ \\
\hline IODINE & $0 \%$ & $58 \%$ & $0 \%$ & $0 \%$ & $36 \%$ & $0 \%$ & $25 \%$ & $31 \%$ & $0 \%$ & $58 \%$ \\
\hline POTASSIUM & $124 \%$ & $212 \%$ & $67 \%$ & $253 \%$ & $144 \%$ & $94 \%$ & $206 \%$ & $217 \%$ & $201 \%$ & $238 \%$ \\
\hline CALCIUM & $53 \%$ & $90 \%$ & $77 \%$ & $179 \%$ & $111 \%$ & $65 \%$ & $84 \%$ & $118 \%$ & $182 \%$ & $99 \%$ \\
\hline MAGNESIUM & $51 \%$ & $124 \%$ & $44 \%$ & $175 \%$ & $73 \%$ & $82 \%$ & $140 \%$ & $207 \%$ & $119 \%$ & $156 \%$ \\
\hline PHOSPHORUS & $105 \%$ & $169 \%$ & $138 \%$ & $175 \%$ & $248 \%$ & $89 \%$ & $113 \%$ & $411 \%$ & $218 \%$ & $249 \%$ \\
\hline$\overline{Z I N C}$ & $35 \%$ & $55 \%$ & $48 \%$ & $124 \%$ & $97 \%$ & $35 \%$ & $78 \%$ & $144 \%$ & $67 \%$ & $164 \%$ \\
\hline SELENIUM & $30 \%$ & $44 \%$ & $24 \%$ & $159 \%$ & $97 \%$ & $3 \%$ & $120 \%$ & $256 \%$ & $117 \%$ & $174 \%$ \\
\hline $\begin{array}{l}\text { INDIVIDUAL } \\
\text { MICRONUTRIENT } \\
\text { DEFICIENCIES } \\
\end{array}$ & 14 & 7 & 15 & 3 & 9 & 13 & 5 & 4 & 7 & 3 \\
\hline
\end{tabular}

\begin{tabular}{|c|c|c|c|c|c|c|c|c|c|c|}
\hline \multicolumn{11}{|c|}{$\begin{array}{c}\text { TABLE } 4 . \\
\text { Group II (5 MEN, } 5 \text { WOMEN) }\end{array}$} \\
\hline MICRONUTRIENT & \multicolumn{5}{|c|}{$\begin{array}{l}\text { MEN \% } \\
\text { Reference Daily Intake (RDI) }\end{array}$} & \multicolumn{5}{|c|}{$\begin{array}{l}\text { WOMEN \% } \\
\text { Reference Daily Intake (RDI) }\end{array}$} \\
\hline $\begin{array}{l}\text { MALE (M) } \\
\text { WOMEN (W) }\end{array}$ & $\begin{array}{c}\text { M6 } \\
(\mathrm{S})\end{array}$ & $\begin{array}{l}\text { M7 } \\
\text { (A) }\end{array}$ & $\begin{array}{l}\text { M8 } \\
\text { (A) }\end{array}$ & $\begin{array}{l}\text { M9 } \\
\text { (A) }\end{array}$ & $\begin{array}{l}\text { M10 } \\
\text { (A) }\end{array}$ & $\begin{array}{l}\text { W6 } \\
\text { (A) }\end{array}$ & $\begin{array}{l}\text { W7 } \\
\text { (S) }\end{array}$ & $\begin{array}{l}\mathrm{W} 8 \\
(\mathrm{~S})\end{array}$ & $\begin{array}{l}\text { W9 } \\
\text { (S) }\end{array}$ & $\begin{array}{l}\text { W10 } \\
(\mathrm{S})\end{array}$ \\
\hline $\begin{array}{l}\text { TOTAL CALORIES } \\
\text { INTAKE REPORTED }\end{array}$ & $42 \%$ & $161 \%$ & $56 \%$ & $93 \%$ & $134 \%$ & $125 \%$ & $118 \%$ & $76 \%$ & $104 \%$ & $64 \%$ \\
\hline VITAMIN A & $248 \%$ & $445 \%$ & $105 \%$ & $129 \%$ & $117 \%$ & $533 \%$ & $318 \%$ & $326 \%$ & $216 \%$ & $130 \%$ \\
\hline VITAMIN D & $75 \%$ & $30 \%$ & $66 \%$ & $13 \%$ & $125 \%$ & $86 \%$ & $43 \%$ & $60 \%$ & $6 \%$ & $16 \%$ \\
\hline VITAMIN E & $1 \%$ & $76 \%$ & $0.2 \%$ & $123 \%$ & $93 \%$ & $19 \%$ & $195 \%$ & $9 \%$ & $173 \%$ & $39 \%$ \\
\hline VITAMIN K & $205 \%$ & $122 \%$ & $104 \%$ & $95 \%$ & $73 \%$ & $341 \%$ & $110 \%$ & $197 \%$ & $189 \%$ & $28 \%$ \\
\hline VITAMIN B-1 & $101 \%$ & $290 \%$ & $95 \%$ & $137 \%$ & $187 \%$ & $255 \%$ & $206 \%$ & $98 \%$ & $176 \%$ & $155 \%$ \\
\hline VITAMIN B-2 & $100 \%$ & $209 \%$ & $106 \%$ & $167 \%$ & $189 \%$ & $219 \%$ & $191 \%$ & $113 \%$ & $143 \%$ & $84 \%$ \\
\hline VITAMIN B-3 & $119 \%$ & $294 \%$ & $126 \%$ & $130 \%$ & $198 \%$ & $204 \%$ & $207 \%$ & $163 \%$ & $186 \%$ & $167 \%$ \\
\hline VITAMIN B-6 & $97 \%$ & $137 \%$ & $73 \%$ & $117 \%$ & $167 \%$ & $168 \%$ & $141 \%$ & $125 \%$ & $133 \%$ & $128 \%$ \\
\hline VITAMIN B-12 & $146 \%$ & $193 \%$ & $179 \%$ & $216 \%$ & $224 \%$ & $111 \%$ & $191 \%$ & $182 \%$ & $161 \%$ & $98 \%$ \\
\hline FOLATE & $151 \%$ & $255 \%$ & $137 \%$ & $190 \%$ & $260 \%$ & $327 \%$ & $156 \%$ & $131 \%$ & $209 \%$ & $171 \%$ \\
\hline
\end{tabular}




\begin{tabular}{|l|l|l|l|l|l|l|l|l|l|l|}
\hline IODINE & $0 \%$ & $0 \%$ & $18 \%$ & $0 \%$ & $0 \%$ & $0 \%$ & $0 \%$ & $0 \%$ & $0 \%$ & $0 \%$ \\
\hline POTASSIUM & $136 \%$ & $253 \%$ & $99 \%$ & $137 \%$ & $260 \%$ & $206 \%$ & $135 \%$ & $171 \%$ & $122 \%$ & $73 \%$ \\
\hline CALCIUM & $59 \%$ & $179 \%$ & $77 \%$ & $127 \%$ & $193 \%$ & $178 \%$ & $95 \%$ & $103 \%$ & $114 \%$ & $69 \%$ \\
\hline MAGNESIUM & $75 \%$ & $175 \%$ & $72 \%$ & $92 \%$ & $146 \%$ & $167 \%$ & $125 \%$ & $110 \%$ & $130 \%$ & $77 \%$ \\
\hline PHOSPHORUS & $119 \%$ & $321 \%$ & $135 \%$ & $193 \%$ & $254 \%$ & $213 \%$ & $148 \%$ & $149 \%$ & $144 \%$ & $65 \%$ \\
\hline ZINC & $41 \%$ & $124 \%$ & $71 \%$ & $68 \%$ & $96 \%$ & $98 \%$ & $98 \%$ & $67 \%$ & $63 \%$ & $56 \%$ \\
\hline SELENIUM & $55 \%$ & $159 \%$ & $47 \%$ & $74 \%$ & $122 \%$ & $125 \%$ & $85 \%$ & $173 \%$ & $146 \%$ & $115 \%$ \\
\hline \begin{tabular}{l} 
INDIVIDUAL $\begin{array}{l}\text { MICRONUTRIENT } \\
\text { DEFICIENCIES }\end{array}$ \\
\hline
\end{tabular} & 8 & 3 & 10 & 6 & 4 & 4 & 5 & 5 & 3 & 11 \\
\hline
\end{tabular}

\begin{tabular}{|c|c|c|c|}
\hline \multicolumn{4}{|c|}{ TABLE 5. MICRONUTRIENT DEFICITS } \\
\hline MICRONUTRIENT & $\begin{array}{c}\text { MICRONUTRIENT } \\
\text { RDA \% }\end{array}$ & MEN \# DEFICIT & WOMEN \# DEFICIT \\
\hline IODINE * & $100 \%$ & 10 & 10 \\
\hline VITAMIN D & $95 \%$ & 9 & 10 \\
\hline ZINC & $80 \%$ & 8 & 8 \\
\hline VITAMIN E & $65 \%$ & 8 & 5 \\
\hline CALORIES & $50 \%$ & 6 & 4 \\
\hline CALCIUM & $50 \%$ & 5 & 5 \\
\hline SELENIUM & $45 \%$ & 7 & 2 \\
\hline VITAMIN K & $45 \%$ & 5 & 4 \\
\hline MAGNESIUM & $40 \%$ & 6 & 2 \\
\hline VITAMIN B-6 & $30 \%$ & 5 & 1 \\
\hline VITAMIN B-2 & $25 \%$ & 3 & 2 \\
\hline VITAMIN A & $25 \%$ & 3 & 2 \\
\hline VITAMIN B-1 & $20 \%$ & 2 & 2 \\
\hline VITAMIN B-12 & $20 \%$ & 1 & 3 \\
\hline POTASSIUM & $20 \%$ & 2 & 2 \\
\hline FOLATE & $15 \%$ & 2 & 1 \\
\hline VITAMIN B-3 & $10 \%$ & 2 & 0 \\
\hline PHOSPHORUS & $10 \%$ & 0 & 2 \\
\hline
\end{tabular}

* Iodine is present in sea vegetation, but not in most foods unless iodized salt is added. An iodine intake of less than 20 micrograms (ug) per day is considered severe deficiency; 20-50 micrograms (ug) per day.

\section{REFERENCES}

1. American Dietetic Association. Position of the American Dietetic Association: fortification and nutritional supplements. J Am Diet Assoc. 2005 Aug;105(8):1300-11. 